\title{
DISCRETE REPRESENTATIONS OF SECOND ORDER RANDOM FUNCTIONS. I
}

UDC 519.21

\author{
O. I. PONOMARENKO
}

\begin{abstract}
We study representations of both scalar and vector random functions defined on a set $T$ in the form of either infinite or finite sums whose terms are scalar functions on $T$ with random coefficients. The assumptions imposed on the set $T$ and on properties of random functions are rather general. In particular, we consider the cases where $T$ is a compact topological space, measurable space with positive measure, or an arbitrary nonempty set. Various examples of such representations are given for specific random functions assuming values in Hilbert spaces. The KarhunenLoeve type representations are considered in part I of the paper. More general basis type representations are studied in part II.
\end{abstract}

\section{INTRODUCTION}

A representation of a second order random function defined on a set $T$ that is written in the form of either a finite or infinite series whose terms are scalar functions on $T$ and whose coefficients are random variables is called a discrete representation. From the point of view of applications, the most important are the representations whose coefficients are orthogonal random variables if such a representation is unique for a given random function.

Several representations of such type become classical in the modern theory of second order stochastic processes and random fields. The Karhunen-Loeve representation of scalar stochastic processes defined in an interval belongs to this type of representations (see [1]-5]) and is based on a representation of the correlation kernel of the process in terms of eigen-functions of the corresponding integral operator known as the Mercer theorem in the theory of integral equations; see [6]. The Kotel'nikov-Shannon type representations [2], various basis type representations related to expansions of functions involved in factorization decompositions of correlation kernels of random functions in the appropriate function spaces, as well as those corresponding to expansions of a random function with respect to a specific basis (see [5, 7]) are also of this type of representations.

In general, discrete representations is a tool of a constructive description of random functions which is important for their modelling [5].

The aim of this paper is to show that the discrete representations of second order random functions mentioned above are valid for a wider class of random functions and for a wider class of their arguments. The Karhunen-Loeve type representations are extended in Section 2 to the class of one-dimensional and multi-dimensional random functions assuming values in Hilbert spaces in the case where their arguments belong to

2010 Mathematics Subject Classification. Primary 60G10, 60G15; Secondary 60G57.

Key words and phrases. Random functions defined on a compact topological space, generalized random functions assuming values in a Hilbert space, Karhunen-Loeve type representations, basis type representations, Hilbert-Schmidt operators. 
a compact set of a topological spaces. Section 3 is devoted to more general basis type representations of random functions related to various factorization bilinear expansions of positive definite kernels known in function analysis (see [8, 9]). In particular, we use those expansions that are based on some results in harmonic analysis in abstract groups, semigroups, etc. concerning the Hilbert-Schmidt operators. This approach allows us to obtain discrete representations of second order random functions under rather general assumptions imposed on the set of arguments of a random function. Following this approach, we link properties of random functions with those of terms of the corresponding representations.

Below we exhibit several examples of how to apply general results for obtaining some important discrete representations of basic stochastic processes and random fields assuming values in Hilbert spaces. In particular, we study stochastic processes and random fields that are spectral nonexpandable from the point of view of the general theory of stationary stochastic processes on Abel involutive semigroups (see [10]).

\section{Karhunen-Loeve type Discrete Representations}

2.1. First we consider representations of one-dimensional second order random functions on compact spaces.

Let $\xi(t), t \in T$, be a complex valued second order random function defined in a probability space $(\Omega, \mathcal{F}, \mathrm{P}), \xi(t) \in L_{2}(\Omega)=L_{2}(\Omega, \mathcal{F}, \mathrm{P})$ (that is, $\mathrm{E}|\xi(t)|^{2}<\infty$ for all $t \in T$, where $T$ is an arbitrary nonempty set. Denote by $m(t), t \in T$, the mean function of $\xi(t)$,

$$
m(t)=\mathrm{E} \xi(t), \quad t \in T,
$$

and by $k(t, s), t, s \in T$, the correlation kernel of the random function $\xi(t)$,

$$
k(t, s)=\mathrm{E} \xi(t) \overline{\xi(s)}, \quad t, s \in T .
$$

The necessary and sufficient condition that a complex function of the two arguments,

$$
k(t, s), \quad t, s \in T
$$

is a correlation kernel of a random function $\xi(t), t \in T$, is that $k(t, s)$ is positive definite. If $k(t, s)$ is a positive definite kernel and a function $m(t)$ is given, then there exists a Gaussian random function $\xi(t), t \in T$, for which $k(t, s)$ is the correlation kernel and $m(t)$ is the mean function (see [1]- 3]).

Let $\{\xi(t), t \in T\}$ be a set of random variables. Denote by $L_{2}(\xi)$ the closure in $L_{2}(\Omega)$ of the linear span of $\{\xi(t), t \in T\}$. Then $L_{2}(\xi)$ is called the space of values of the random function $\xi(t)$.

Let $T$ be a compact topological space, let $\mathcal{B}=\mathcal{B}(T)$ be the $\sigma$-algebra of Borel sets in the space $T$, and let $\xi(t), t \in T$, be a mean square continuous random function on the space $T$. Then the correlation kernel $k(t, s)$ of the random function $\xi(t)$ is continuous in the topological product $T \times T$. The criteria of the continuity of $\xi(t)$ expressed in terms of the correlation kernel is that $\operatorname{Re} k(t, s)$ is continuous on the diagonal of the square $T \times T$ (see [1]- 3], 8]).

Denote by $\mu$ a $\sigma$-additive nonnegative finite measure in $\mathcal{B}(T)$ such that $\mu(V)>0$ for all open sets $V, V \subset T$. If $T$ is a compact set in a local compact group $G$, then the restriction of the Haar measure in $G$ is often taken for $\mu$. For the cases, where $G$ is the $n$-dimensional Euclidean space, that is, where $G=\mathbf{R}^{n}$, the Lebesgue measure in $T$ is taken as the measure $\mu$. 
Consider the integral operator $K$ generated by the kernel $k(t, s)$. This operator acts in the space of functions $\varphi(t)$ belonging to $L_{2}(T, \mathcal{B}, \mu)$ as follows:

$$
K \varphi(t)=\int_{T} k(t, s) \varphi(s) \mu(d s), \quad t \in T
$$

Note that this operator is completely continuous, selfadjoint, and positive.

Let $\left\{\varphi_{j}(t)\right\}, j \in J$, be a complete orthonormal system of eigenfunctions of the operator $K$. Let $\left\{\varphi_{j}(t)\right\}$ correspond to the system of eigenvalues $\left\{\lambda_{j}\right\}, \lambda_{j}>0$, of the operator $K$, that is,

$$
\begin{gathered}
K \varphi_{j}(t)=\lambda_{j} \varphi_{j}(t), \quad j \in J, \\
\int_{T} \varphi_{j}(t) \varphi_{r}(t) \mu(d t)=\delta_{i j}= \begin{cases}1, & j \neq r, \\
0, & j=r .\end{cases}
\end{gathered}
$$

The following generalization of the Mercer theorem (see [9]), belongs to Krĕn (see [8]): if the above assumptions hold, then

$$
k(t, s)=\sum_{j \in J} \lambda_{j} \varphi_{j}(t) \overline{\varphi_{j}(s)}, \quad t, s \in T .
$$

Equality (2.5) is called the bilinear representation of the kernel $k$. If the set $J$ is infinite, then the series in (2.5) converges absolutely and uniformly in $T \times T$ and the functions $\varphi_{j}(t)$ are $\omega$-independent in the Krel̆ sense. The latter notion means that if

$$
\sum_{j \in J} c_{j} \varphi_{j}(t)=0, \quad t \in T, \quad \sum_{j \in J}\left|c_{j}\right|^{2}<\infty, c_{j} \in \mathbf{C},
$$

then $c_{j}=0$ for all $j$ (here the symbol $\mathbf{C}$ stands for the set of complex numbers). Moreover, every series $\sum_{j \in J} c_{j} \sqrt{\lambda_{j}} \varphi_{j}(t)$ uniformly converges if the coefficients $c_{j}, j \in J$, satisfy the second condition in (2.6). Different measures $\mu$ in the definition (2.2) of the operator $K$ correspond to different systems of $\omega$-independent functions $\left\{\varphi_{j}(t)\right\}, j \in J$. Every system $\left\{\varphi_{j}(t)\right\}, j \in J$, can be obtained from another one with the help of a unitary transformation.

Moreover, $K$ is a kernel operator acting according to the rule

$$
K \psi(t)=\sum_{j \in J} \lambda_{j}\left(\psi \mid \varphi_{j}\right) \varphi_{j}, \quad \psi \in L_{2}(T, \mathcal{B}, \mu)
$$

where $(\cdot \mid \cdot)$ is the scalar product, and the $\operatorname{trace} \operatorname{tr} K$ is such that

$$
\operatorname{tr} K=\int_{T} k(s, s) \mu(d s) .
$$

Theorem 2.1. Let $\xi(t), t \in T$, be a mean square continuous random function defined on a compact space $T$. Then $\xi(t), t \in T$, admits the representation

$$
\xi(t)=\sum_{j \in J} \sqrt{\lambda_{j}} \varphi_{j}(t) z_{j}, \quad t \in T
$$

where $\left\{z_{j}, j \in J\right\}$ is a system of orthonormal random variables in $L_{2}(\xi)$ such that

$$
z_{j}=\frac{1}{\sqrt{\lambda_{j}}} \int_{T} \xi(t) \overline{\varphi_{j}(t)} \mu(d t), \quad j \in J,
$$

and where $\left\{\varphi_{j}(t)\right\}_{j \in J}$ and $\left\{\lambda_{j}\right\}_{j \in J}$ are the systems of eigenfunctions and eigennumbers of the integral operator $K$ constructed from the correlation kernel $k(t, s)$ of the function $\xi(t)$ according to equality (2.2). If the set $J$ is infinite, then the series (2.7) converges uniformly in the mean square sense. 
If $\xi(t)$ is a real-valued zero mean Gaussian random function, then the coefficients $z_{j}$ in representation (2.7) are independent Gaussian random variables and the series in (2.7) converges with probability one for all $t \in T$.

Proof. According to the results mentioned above, the correlation kernel $k(t, s)$ of the random function $\xi(t)$ admits bilinear representation (2.5) which can be treated as the integral representation in the Karhunen theorem (see [3]) with the counting measure in the set $J$. The Karhunen theorem implies representation (2.8). The uniform convergence of the series in (2.7) in the strong topology of $L_{2}(\Omega)$ follows from the uniform convergence of the series in (2.5).

The integrals in (2.8) are understood in the sense of Bochner integrals of vector-valued functions $\xi(t) \overline{\varphi_{j}(t)}$ with values in the Hilbert space $L_{2}(\Omega)$. The integrals on the righthand side of (2.8) exist, since

$$
\int_{T}\left|\varphi_{j}(t)\right| \cdot\|\xi(t)\|_{L_{2}(\Omega)} \mu(d t)<\infty
$$

(see [11]). Substituting representation (2.7) to the integral in (2.8) we obtain equality (2.8), since the functions $\varphi_{j}$ are orthonormal in view of (2.4).

If $\xi(t)$ is a real-valued zero mean Gaussian random function, then the space $L_{2}(\xi)$ consists of Gaussian zero mean random variables. This implies that the coefficients $z_{j}$, $j \in J$, in representation (2.7) are uncorrelated Gaussian random variables, whence we conclude that they are independent. Finally, since the series

$$
\sum_{j \in J} \mathrm{E}\left(\sqrt{\lambda_{j}} \varphi_{j}(t) z_{j}\right)^{2}=\sum_{j \in J} \lambda_{j}\left|\varphi_{j}(t)\right|^{2}=k(t, t)
$$

converges, the series in (2.7) converges with probability one for all $t \in T$.

Now we are going to exhibit some applications of representation (2.7) for solving some main approximation problems for random functions.

The problem of optimal linear prediction of second order random functions $\xi(v), v \in V$, in a topological space $V$ from observations $\{\xi(t), t \in T\}$, where $T$ is a compact subset of the space $V$, is to find the estimate $\hat{\xi}(v)$ for $\xi(v), v \notin T$, that is the best in the sense of the mean square error and belongs to the closure $L_{2}^{T}(\xi)$ in $L_{2}(\Omega)$ of the linear span of the set of observations.

The problem of optimal linear filtration constructed from observations $\{\xi(t), t \in T\}$ is to find the best linear estimate $\hat{\eta}(v)$ of a second order random function $\eta(v), v \in V$, defined in the same probability space where $\xi(v)$ is defined.

Assume that the function $\xi(v)$ is mean square continuous in $T$. Assume further that the correlation functions $k(v, t)=\mathrm{E} \xi(v) \overline{\xi(t)}, t \in T$, and $\tilde{k}(v, t)=\mathrm{E} \eta(v) \overline{\xi(t)},(v, t) \in$ $V \times T$, as well as the eigenfunctions and eigennumbers of the integral operator with the kernel $k(t, s)=\mathrm{E} \xi(t) \overline{\xi(s)}, t, s \in T$, are known.

Theorem 2.2. Under the above assumptions, the best linear prediction of $\xi(v), v \notin T$, is given by

$$
\hat{\xi}(v)=\sum_{j \in J} \frac{1}{\lambda_{j}}\left(\int_{T} k(v, t) \varphi_{j}(t) \mu(d t)\right)\left(\int_{T} \xi(t) \overline{\varphi_{j}(t)} \mu(d t)\right),
$$

while the best linear estimate $\hat{\eta}(v), v \in V$, for the problem of filtration is given by

$$
\hat{\eta}(v)=\sum_{j \in J} \frac{1}{\lambda_{j}}\left(\int_{T} \tilde{k}(v, t) \varphi_{j}(t) \mu(d t)\right)\left(\int_{T} \xi(t) \overline{\varphi_{j}(t)} \mu(d t)\right) .
$$


Proof. We prove the equality for the best linear prediction. Representation (2.7) implies that the random variables $z_{j}$ form an orthonormal basis in the space of observations $L_{2}^{T}(\xi)$. The estimate $\hat{\xi}(v)$ is the orthogonal projection in $L_{2}(\xi)$ onto the subspace $L_{2}^{T}(\xi)$. Thus $\hat{\xi}(v)$ is of the form

$$
\hat{\xi}(v)=\sum_{j \in J} a_{j}(v) z_{j}, \quad a_{j}(v)=\mathrm{E} \hat{\xi}(v) \bar{z}_{j} .
$$

The difference $\xi(v)-\hat{\xi}(v)$ is orthogonal to $L_{2}^{T}(\xi)$, whence we conclude that

$$
a_{j}(v)=\mathrm{E} \xi(v) \bar{z}_{j}=\frac{1}{\sqrt{\lambda}_{j}} \int_{T} k(v, t) \varphi_{j}(t) \mu(d t), \quad j \in J .
$$

This together with (2.8) implies the result desired.

The equality for the problem of filtration is proved similarly.

Note that the results of this section extend those obtained by the author in [20].

2.2. Consider the Karhunen-Loeve type representation for random functions assuming values in Hilbert spaces.

Let $H$ be a complex Hilbert space and let $\mathcal{L}\left(H, L_{2}(\Omega)\right)$ be the Banach space of all linear continuous operators $\Xi$ acting from $H$ to $L_{2}(\Omega)$. We consider the usual operator norm.

Every element $\Xi \in \mathcal{L}\left(H, L_{2}(\Omega)\right)$ is a random $L_{2}(\Omega)$-valued linear continuous functional in $H$. One can treat $\Xi$ as a generalized random element in $H$. The usual $H$-valued random element $\xi(\omega), \omega \in \Omega$, such that $\mathrm{E}\|\xi\|^{2}<\infty$, generates a unique functional $\Xi$ by $\Xi x=(x \mid \xi)_{H}$. We identify $\Xi$ with $\xi$ and call it a regular generalized random element in $H$.

In general, $\Xi \in \mathcal{L}\left(H, L_{2}(\Omega)\right)$ can be treated as the usual random element in a certain extension of the space $H$ (see [12]). If the space $H$ is finite dimensional, then each element $\Xi x=(x \mid \xi)_{H}$ is regular. In the general case, an element $\Xi$ is regular if and only if $\Xi$, considered as an operator of $\mathcal{L}\left(H, L_{2}(\Omega)\right)$, has a finite Hilbert-Schmidt norm.

The expectation $m=\mathrm{E} \Xi$ of an element $\Xi \in \mathcal{L}\left(H, L_{2}(\Omega)\right)$ is defined as a vector $m \in H$ such that $\mathrm{E}(\Xi x)=(x \mid m), x \in H$. The correlation operator $[\Xi, \Psi]$ for $\Xi, \Psi \in \mathcal{L}\left(H, L_{2}(\Omega)\right)$ is defined as an element of the Banach algebra $\mathcal{B}(H)$ of all linear continuous operators in $H$ such that

$$
([\Xi, \Psi] x \mid y)=\mathrm{E}(\Xi x) \overline{(\Psi y)}, \quad x, y \in H .
$$

It is obvious that $[\Xi, \Psi]=\Psi^{*} \Xi$, where $\Psi^{*} \in \mathcal{L}\left(H, L_{2}(\Omega)\right)$ is the dual operator to $\Psi$ such that

$$
\left(\Psi^{*} \eta \mid x\right)_{H}=(\eta \mid \Psi x)_{L_{2}(\Omega)}, \quad x \in H, \eta \in L_{2}(\Omega) .
$$

Note that $[\Xi, \Psi]$ is a sesquilinear form in $\mathcal{L}\left(H, L_{2}(\Omega)\right)$ such that $[\Xi, \Xi]$ belongs to the convex cone $\mathcal{B}_{+}(H)$ of positive Hermitian operators of $\mathcal{B}(H)$. Also, $\Xi$ is regular if $[\Xi, \Xi]$ is a kernel operator. The set $\mathcal{L}\left(H, L_{2}(\Omega)\right)$ of generalized random elements in $H$ possesses the structures of a right unitary module over $\mathcal{B}(H)$ and left unitary module over $\mathcal{B}\left(L_{2}(\Omega)\right)$.

Every family of random elements $\left\{\Xi_{t} \in \mathcal{L}\left(H, L_{2}(\Omega)\right), t \in T\right\}$ is called a generalized second order random function $\Xi_{t}, t \in T$, which is defined on the set $T$ and that assumes values in the space $H$. Denote by $m(t), t \in T$, the mean function of $\Xi_{t}, m(t)=\mathrm{E} \Xi_{t} \in H$. The correlation kernel of $\Xi_{t}$ is denoted by

$$
R(s, t)=\Xi_{s}^{*} \Xi_{t}, \quad t, s \in T, \quad R(t, s) \in \mathcal{B}(H) .
$$

A random function $\Xi_{t}, t \in T$, is called Gaussian if $\Xi_{t} x=\xi(t, x)$ is a complex-valued Gaussian function in $T \times H$. 
The $\mathcal{B}(H)$-valued operator kernel $R(s, t), t, s \in T$, is a correlation kernel for some random function $\Xi_{t}, t \in T$, assuming values in $H$ if and only if $R(s, t)$ is positive definite; that is,

$$
\sum_{j=1}^{n} \sum_{r=1}^{n}\left(R\left(t_{i}, t_{r}\right) x_{j} \mid x_{r}\right) \geq 0
$$

for all positive integer numbers $n$ and for all $x_{j} \in H$ and $t_{j} \in T, j=1, \ldots, n$. If $R(s, t)$ is a correlation kernel and $m(t)$ is a function, then there exists a Gaussian random function $\Xi_{t}, t \in T$, that assumes values in $H$, has the correlation kernel $R(t, s)$, and whose mean function is $m(t)$.

Theorem 2.3. Assume that $\Xi_{t}, t \in T$, is a generalized second order random function defined on a compact space $T$ and that assumes values in $H$. Let its correlation kernel be given by $R(t, s)=k(t, s) A$, where $A \in \mathcal{B}_{+}(H)$ is an operator and $k(t, s)$ is a positive definite kernel in $T \times T$. Then

$$
\Xi_{t}=\sum_{j \in J} \sqrt{\lambda_{j}} \varphi_{j}(t) \Phi_{j}, \quad t \in T
$$

where $\left\{\varphi_{j}(t), j \in J\right\}$ is an orthonormal system of eigenfunctions corresponding to eigennumbers $\left\{\lambda_{j}, j \in J\right\}$ of the integral operator $K$ generated by the kernel $k(t, s)$ and where $\left\{\Phi_{j}, j \in J\right\}$ are random elements that belong to the space $\mathcal{L}\left(H, L_{2}(\Omega)\right)$ and such that

$$
\Phi_{r}^{*} \Phi_{j}=\delta_{r j} A .
$$

If the set $J$ is infinite, then the series on the right-hand side of representation (2.9) converges uniformly in $T$ with respect to the norm in $\mathcal{L}\left(H, L_{2}(\Omega)\right)$. If $A$ is a kernel operator, then the random elements $\left\{\Phi_{j}, j \in J\right\}$ are regular and the function $\Xi_{t}$ is regular as well (the latter notion means that $\Xi_{t}$ can be identified with a usual random function $\xi(t)$ assuming values in the space $H$ and such that $\mathrm{E}\|\xi(t)\|^{2}<\infty$ for all $\left.t \in T\right)$.

Proof. The results obtained in Section 2.1 imply that the kernel $R(t, s)$ admits the representation

$$
R(t, s)=\sum_{j \in J} \lambda_{j} \varphi_{j}(t) \bar{\varphi}_{j}(s) A, \quad t, s \in T,
$$

where the series on the right-hand side converges with respect to the norm in $\mathcal{B}(H)$ and uniformly in two arguments $t, s$. Then we use Theorem 2 of the paper [13] on a representation of generalized random functions in vector topological space, namely we apply Theorem 2 of 13 to representation (2.10) and obtain representation (2.9). The convergence of series on the right-hand side of (2.10) discussed above implies that this series converges uniformly in $T$ with respect to the norm in $\mathcal{L}\left(H, L_{2}(\Omega)\right)$.

Since $A$ is a kernel operator, the random elements $\Phi_{j}$ are regular and the function $\Xi_{t}$ is regular, as well.

2.3. Now we are going to exhibit some applications of Theorem 2.2 and discuss some specific classes of random functions assuming values in $H$. The scalar case is a particular case corresponding to $\operatorname{dim} H=1$.

Example 2.1. Let $\Xi_{t}$ be a stochastic process in the space $H$ defined in the interval $[0, \ell]$ and whose correlation kernel is given by

$$
R(t, s)=[\min (t, s)] A, \quad A \in \mathcal{B}_{+}(H), t, s \in[0, \ell] \subset \mathbf{R}_{+} .
$$

Thus the increments of the stochastic process $\Xi_{t}$ are orthogonal, that is,

$$
\left(\Xi_{t_{4}}-\Xi_{t_{3}}\right) *\left(\Xi_{t_{2}}-\Xi_{t_{1}}\right)=0
$$

for $0 \leq t_{1}<t_{2} \leq t_{3}<t_{4} \leq \ell$. 
If the spaces $H$ and $L_{2}(\Omega)$ are real and if $\Xi_{t}$ is a zero mean Gaussian process, then $\Xi_{t}$ is a generalized Brownian motion in $H$ (in other words, $\Xi_{t}$ is a Wiener process). If $A=I$, where $I$ is a unit operator, then $\Xi_{t}$ is a standard Brownian motion in $H$.

The eigenfunctions and eigennumbers of the integral operator with the kernel

$$
\min (t, s)
$$

on the interval $[0, \ell]$ are given by

$$
\begin{gathered}
\tilde{\varphi}_{j}(t)=\sin \frac{(2 j+1) \pi t}{2 \ell}, \quad \lambda_{j}=\frac{4 \ell^{2}}{[(2 j+1) \pi]^{2}}, \\
\left\|\tilde{\varphi}_{j}\right\|_{L_{2}[0, \ell]}^{2}=\frac{\ell}{2}, \quad j=0,1,2, \ldots,
\end{gathered}
$$

(see [14]). The functions $\varphi_{j}(t)=\sqrt{2 / \ell} \cdot \tilde{\varphi}_{j}(t)$ are orthonormal. Thus Theorem2.2 implies the representation

$$
\Xi_{t}=\frac{2 \sqrt{2 \ell}}{\pi} \sum_{j=0}^{\infty} \frac{\sin \frac{(2 j+1) \pi t}{2 \ell}}{(2 j+1)} \Phi_{j}, \quad t \in[0, \ell],
$$

where $\Phi_{j} \in \mathcal{L}\left(H, L_{2}(\Omega)\right)$ and $\Phi_{r}^{*} \Phi_{j}=\delta_{r j} A$.

Note that there is a straightforward proof of representation (2.12). The proof is based on the expansion of the function $g(t)=\min (t, s)$ with respect to the sinuses in the interval $[0, \ell]$ if $s$ is fixed. Thus,

$$
R(t, s)=\frac{8 \ell}{\pi^{2}} \sum_{j=0}^{\infty} \frac{\sin \frac{(2 j+1) \pi t}{2 \ell} \sin \frac{(2 j+1) \pi s}{2 \ell}}{(2 j+1)^{2}} A,
$$

whence representation (2.12) follows by Theorem 2 of the paper [13].

Example 2.2. Let $\Xi_{t}, t \in[0, \ell]$, be a stochastic process in the space $H$ whose correlation kernel is given by

$$
R(t, s)=\ell^{-1}[\ell \min (t, s)-t s] A, \quad A \in \mathcal{B}_{+}(H) .
$$

Then $\Xi_{0}=\Xi_{\ell}=0$. The stochastic process $\Xi_{t}$ is called the stochastic bridge in $[0, \ell]$. If $\Xi_{t}$ is a zero mean Gaussian process, then $\Xi_{t}$ is a Brownian bridge. If $\ell=1$, then the Brownian bridge $\Xi_{t}$ can be represented as the process $W_{t}-t W_{1}$, where $W_{t}$ is the Brownian motion in $[0,1]$.

The eigenfunctions and eigennumbers of the integral operator corresponding to the kernel $\ell^{-1}[\ell \min (t, s)-t s]$ are given by

$$
\tilde{\varphi}_{j}(t)=\sin \frac{j \pi t}{\ell}, \quad \lambda_{j}=\frac{\pi^{2} j^{2}}{\ell^{2}}, \quad\left\|\tilde{\varphi}_{j}\right\|_{L_{2}[0, \ell]}^{2}=\frac{\ell}{2}, \quad j=1,2, \ldots
$$

(see [15]).

Then Theorem 2.2 implies that

$$
\begin{gathered}
\Xi_{t}=\frac{\sqrt{2 \ell}}{\pi} \sum_{j=1}^{\infty} \frac{\sin \frac{j \pi t}{\ell}}{j} \Phi_{j}, \quad t \in[0, \ell], \\
R(t, s)=\frac{2 \ell}{\pi^{2}} \sum_{j=1}^{\infty} \frac{\sin \frac{j \pi t}{\ell} \sin \frac{j \pi s}{\ell}}{j^{2}} A
\end{gathered}
$$

where $\Phi_{j} \in \mathcal{L}\left(H, L_{2}(\Omega)\right), \Phi_{r}^{*} \Phi_{j}=\delta_{r j} A$. 
Example 2.3. Let $\Xi_{t}, t \in[0,1]$, be a stochastic process in the space $H$ whose correlation kernel is given by

$$
R(t, s)=e^{-|t-s|} A, \quad A \in \mathcal{B}_{+}(H) .
$$

Then it is natural to say that $\Xi_{t}$ is a generalized Ornstein-Uhlenbeck process in $H$.

The process $\Xi_{t}$ in $\mathbf{R}=(-\infty, \infty)$ is a stationary process if $\mathbf{E} \Xi_{t}=m=$ const. It admits the following representation

$$
\Xi_{t}=\int_{\mathbf{R}} e^{i \lambda t} \Phi(d \lambda), \quad t \in \mathbf{R}
$$

where $\Phi$ is a $\mathcal{L}\left(H, L_{2}(\Omega)\right)$-valued orthogonal Radon measure in $\mathbf{R}$ such that

$$
\Phi^{*}\left(\Delta_{1}\right) \Phi\left(\Delta_{2}\right)=\left(\frac{1}{\pi} \int_{\Delta_{1} \cap \Delta_{2}} \frac{d \lambda}{1+\lambda^{2}}\right) A
$$

for all Borel sets $\Delta_{1}, \Delta_{2} \subset \mathbf{R}$.

The eigenfunctions $\tilde{\varphi}_{j}$ and eigennumbers $\lambda_{j}$ of the integral kernel $e^{-|t-s|}, t, s \in[0,1]$, are given by

$$
\tilde{\varphi}_{j}(t)=\sin \alpha_{j} t+\alpha_{j} \cos \alpha_{j} t, \quad \lambda_{j}=\frac{1+\alpha_{j}^{2}}{2},
$$

where $\alpha_{j}$ are the roots of the equation $2 \cot \alpha=\alpha-\frac{1}{\alpha}$ (see [14, Example 216]). Moreover,

$$
\left\|\tilde{\varphi}_{j}(t)\right\|_{L_{2}[0,1]}=c_{j}^{2} \sin ^{2} \alpha_{j}, \quad c_{j}^{2}=\frac{3}{4}-\frac{1}{4 \alpha_{j}}+\frac{\alpha_{j}}{4}+\frac{\alpha_{j}^{2}}{2} .
$$

Hence we obtain the representation

$$
\Xi_{t}=\sum_{j}\left(\frac{\sqrt{1+\alpha_{j}^{2}}}{\sqrt{2} c_{j} \sin \alpha_{j}}\right)\left(\sin \alpha_{j} t+\alpha_{j} \cos \alpha_{j} t\right) \Phi_{j}, \quad t \in[0,1],
$$

where $\Phi_{r}^{*} \Phi_{j}=\delta_{r j} A$.

Example 2.4. Let $\Xi_{t}$ be a random field in the space $H$ defined on the $n$-dimensional parallelepiped $M_{\ell}^{n}=\times_{r=1}^{n}\left[0, \ell_{r}\right], \ell=\left(\ell_{1}, \ldots, \ell_{n}\right)$, whose correlation kernel is given by

$$
R(t, s)=\left(\prod_{r=1}^{n} \min \left(t_{r}, s_{r}\right)\right) A=k(t, s) A, \quad A \in \mathcal{B}_{+}(H),
$$

where $t=\left(t_{1}, \ldots, t_{n}\right)$ and $s=\left(s_{1}, \ldots, s_{n}\right)$. The field $\Xi_{t}, t \in M_{\ell}^{n}$, has orthogonal $n$ dimensional increments. If $\Xi_{t}$ is a zero mean Gaussian field, then it is treated as a generalized Chentsov-Wiener field in $H$.

Since the kernel $k(t, s)$ is a product of kernels, the eigenfunctions and eigennumbers of the integral operator generated by $k(t, s)$ are given by

$$
\tilde{\varphi}_{j}(t)=\prod_{r=1}^{n} \sin \frac{\left(2 j_{r}+1\right) \pi t_{r}}{2 \ell_{r}}, \quad \lambda_{j}=\prod_{r=1}^{n} \frac{4 \ell_{r}^{2}}{\left[\left(2 j_{r}+1\right) \pi\right]^{2}}, \quad\left\|\tilde{\varphi}_{j}(t)\right\|^{2}=\frac{\ell_{1} \ell_{2} \ldots \ell_{n}}{2^{n}},
$$

according to results of Example 2.1, where $j=\left(j_{1}, \ldots, j_{n}\right) \in \mathbf{Z}_{+}^{n}$ is a multi-index (here $\mathbf{Z}_{+}$ denotes the set of positive nonnegative numbers).

Then Theorem 2.2 implies the representation

$$
\Xi_{t}=\frac{(2 \sqrt{2})^{n} \sqrt{\ell_{1} \ldots \ell_{n}}}{\pi^{n}} \sum_{j \in Z_{+}^{n}}\left(\prod_{r=1}^{n} \frac{\sin \left(2 j_{r}+1\right) \pi t_{r} / 2 \ell_{r}}{\left(2 j_{r}+1\right)}\right) \Phi_{j}, \quad t \in M_{\ell}^{n},
$$

where $\Phi_{i}^{*} \Phi_{j}=\delta_{i j} A$. 
Example 2.5. Let $\Xi_{t}, t \in M_{\ell}^{n}$, be a random field in the space $H$ whose correlation kernel is given by

$$
R(t, s)=\left(\prod_{r=1}^{n} \ell_{r}^{-1}\left[\ell_{r} \min \left(t_{r}, s_{r}\right)-t_{r} s_{r}\right]\right) A, \quad A \in \mathcal{B}_{+}(H) .
$$

Equality (2.20) yields $\Xi_{t}=0$ for $t \in \partial M_{\ell}^{n}$ (here $\partial M_{\ell}^{n}$ means the boundary of the parallelepiped $M_{\ell}^{n}$ ). This explains the names "stochastic box" for the field $\Xi_{t}$ on $M_{\ell}^{n}$ if $n \geq 3$ and "stochastic sheet" if $n=2$.

Following reasoning similar to that used in Examples 2.4 and 2.1 and taking into account Example 2.2 we obtain the decomposition

$$
\Xi_{t}=\frac{(2)^{n / 2} \sqrt{\ell_{1} \cdots \ell_{n}}}{\pi^{n}} \sum_{j \in \mathbf{N}^{n}}\left(\prod_{r=1}^{n} \frac{1}{j_{r}} \sin \frac{2 j_{r} \pi t_{r}}{\ell_{r}}\right) \Phi_{j}, \quad t \in M_{\ell}^{n},
$$

where $\Phi_{i}^{*} \Phi_{j}=\delta_{i j} A$ and where the symbol $\mathbf{N}$ stands for the set of positive integer numbers.

Example 2.6. Let $\Xi_{t}, t \in \mathbf{R}$, be a stochastic process in $H$ whose correlation kernel is given by

$$
R(t, s)=k(t-s) A, \quad A \in \mathcal{B}_{+}(H),
$$

where $k(t)$ is a real-valued positive definite function. If $\mathrm{E} \Xi_{t}=$ const, then $\Xi_{t}$ is a weakly stationary stochastic process. Consider the process $\Xi_{t}$ in the symmetric interval $[-\pi, \pi]$, where the integral operator $K$ with the kernel $k(t-s)$ has the eigennumbers

$$
\lambda_{n}=\pi a_{n}, \quad a_{n}=\frac{1}{\pi} \int_{-\pi}^{\pi} k(t) \cos n t d t, \quad n=0,1,2, \ldots .
$$

Each eigennumber $\lambda_{n}, n \geq 1$, is multiple and corresponds to two linearly independent eigenfunctions, $\cos n t$ and $\sin n t, n \geq 1$, while $\lambda_{0}$ corresponds to the unique eigenfunction $\varphi_{0}(t) \equiv 1$ (see [14]). According to (2.9), the Fourier representation holds with random orthogonal coefficients, namely

$$
\Xi_{t}=\sqrt{\frac{a_{0}}{2}} \Phi_{0}+\sum_{n=1}^{\infty} \sqrt{a_{n}}\left(\Phi_{n}^{1} \cos n t+\Phi_{n}^{2} \sin n t\right) .
$$

In particular, if $k(t)=\cos ^{2} t$, then the eigennumbers of the operator $K$ are $\lambda_{0}=\pi$ and $\lambda_{2}=\frac{\pi}{2}$ and the corresponding eigenfunctions are $\varphi_{0}(t)=1, \varphi_{2}^{1}(t)=\cos 2 t$, and $\varphi_{2}^{2}(t)=\sin 2 t$. Thus, the right-hand side of (2.22) contains three terms.

2.4. Part II of the present paper contains more general expansions of second order random functions.

\section{BIBLIOGRAPHY}

1. I. I. Gikhman and A. V. Skorokhod, The Theory of Stochastic Processes, vol. 1, "Nauka", Moscow, 1971; English transl., Springer-Verlag, Berlin-Heidelberg, 2004.

2. I. I. Gikhman and A. V. Skorokhod, Introduction to the Theory of Random processes, "Nauka", Moscow, 1977; English transl., Saunders, Philadelphia, 1969. MR.0247660 (40:923)

3. A. V. Bulinskiu and A. N. Shiryaev, Theory of Stochastic Processes, "Fizmatgiz", Moscow, 2003. (Russian)

4. D. V. Gusak, O. G. Kukush, O. M. Kulik, Yu. S. Mishura, and A. Yu. Pilipenko, Theory of Stochastic Processes: With Applications to Financial Mathematics and Risk Theory, Kiev University Press, Kyiv, 2008; English transl., Springer-Verlag, New York, 2010. MR2572942 (2011f:60069)

5. B. V. Dovgă̌, Yu. V. Kozachenko, and I. V. Rozora, Modelling Stochastic Processes in Physical Systems, "Zadruga", Kyiv, 2010. (Ukrainian) 
6. I. G. Petrovskiur, Lectures in the Theory of Integral Equations, "Nauka", Moscow, 1965. (Russian) MR.0352904(50:5390)

7. A. M. Yaglom, Second-order homogeneous random fields, Proc. Fourth Berkeley Symp. Math. Stat. Probab., Vol. II, Univ. Calif. Press, 1961, 593-622. MR0146880 (26:4399)

8. M. G. Krĕn, Hermitian positive kernels in homogeneous spaces. I, Ukr. Mat. Zh. I (1949), no. 4, 64-98. (Russian) MR0051438 (14:480a)

9. F. Riesz and B. Szökefalvi Nagy, Leçons d'analyse fonctionnelle, sixième édition, Akadémiai Kiadó, Budapest, 1972.

10. O. I. Ponomarenko and Yu. D. Perun, Multidimensional weakly stationary random functions on semigroups, Teor. Imovir. Matem. Statyst. 73 (2006), 134-145; English transl. in Theory Probab. Math. Statist. 73 (2005), 151-162. MR2213849 (2007b:60092)

11. E. Hille and R. S. Phillips, Functional Analysis and Semi-Groups, American Mathematical Society, Providence, RI, 1974. (Third printing of the revised edition of 1957, American Mathematical Society Colloquium Publications, Vol. XXXI) MR0423094 (54:11077)

12. O. I. Ponomarenko, Second-order random linear functionals. I, Teor. Imovir. Matem. Statyst. 54 (1996), 137-146; English transl. in Theory Probab. Math. Statist. 54 (1997), 143-151. MR 1644594 (99h:60011)

13. O. I. Ponomarenko, Integral representations of random functions with values in locally convex spaces, Teor. Imovir. Matem. Statyst. 46 (1992), 132-141; English transl. in Theory Probab. Math. Statist. 46 (1993), 129-136. MR.1196216 (93k:60123)

14. M. L. Krasnov, A. I. Kiselev, and G. I. Makarenko Integral Equations, "Nauka", Moscow, 1968. (Russian) MR0265876 (42:785)

15. M. L. Krasnov, Integral Equations, "Nauka", Moscow, 1968. (Russian) MR0511171 (58:23392)

16. N. Dunford and J. T. Schwartz, Linear Operators, Part II. Spectral Theory, Wiley-Interscience, New York-London, 1963. MR.1009163 (90g:47001b)

17. O. Ponomarenko and Yu. Perun, Multivariate random fields on some homogeneous spaces, Theory Stoch. Process. 14(30) (2008), no. 3-4, 104-113. MR2498608 (2010h:60155)

18. N. Ya. Vilenkin, Special Functions and the Theory of Group Representations, "Nauka", Moscow, 1965; English transl., American Mathematical Society, Providence, R. I., 1968. MR1177172 (93d:33013)

19. O. I. Ponomarenko and Yu. D. Perun, Multi-dimensional additively stationary random functions on convex structures, Teor. Imovir. Matem. Statyst. 74 (2006), 118-130; English transl. in Theory Probab. Math. Statist. 74 (2007), 133-146. MR2336784(2008g:60103)

20. A. I. Ponomarenko, Stochastic Problems of Optimization, Kiev University Press, Kiev, 1980. (Russian)

Department of Probability Theory, Statistics, and Actuarial Mathematics, Faculty for Mechanics and Mathematics, National Taras Shevchenko University, Academician Glushkov Avenue, 4E, Kiev 03127, Ukraine

Received 08/SEP/2011

Translated by S. V. KVASKO 